\title{
Mannose shows antitumour properties against lung cancer via inhibiting proliferation, promoting cisplatin-mediated apoptosis and reducing metastasis
}

\author{
YOUYU WANG ${ }^{*}$, SHENGLONG XIE* and BIN HE \\ Department of Thoracic Surgery, Sichuan Academy Medical Sciences and \\ Sichuan Provincial People's Hospital, Chengdu, Sichuan 610000, P.R. China \\ Received February 27, 2019; Accepted July 9, 2019
}

DOI: $10.3892 / \mathrm{mmr} .2020 .11354$

\begin{abstract}
It has been reported that mannose exerts antitumour effects against certain types of cancer. The present study was designed to evaluate whether mannose exerted potential anticancer effects on A549 and H1299 non-small cell lung cancer (NSCLC) cells in vitro, which has not been reported previously. A Cell Counting Kit- 8 cell viability assay was used to assess the antiproliferative effects of mannose on NSCLC cells. Flow cytometry-based methods were used to evaluate the effects of mannose on the cell cycle distribution and cisplatin-mediated apoptosis of NSCLC cells. Transwell migration and invasion assays were conducted to examine whether mannose could inhibit the invasive abilities of NSCLC cells. The effects of mannose on the PI3K/AKT and ERK signalling pathways were explored through western blot analysis assessing the expression of phosphorylated (p)-AKT and p-ERK1/2. It was found that mannose showed potential anticancer effects against NSCLC cells in vitro by inhibiting proliferation, inducing G0/G1 cell cycle arrest, promoting cisplatin-induced apoptosis and decreasing the invasive abilities. These data indicate the potential anticancer properties of mannose and suggest the application of mannose-based therapies to treat NSCLC.
\end{abstract}

Correspondence to: Professor Bin He, Department of Thoracic Surgery, Sichuan Academy Medical Sciences and Sichuan Provincial People's Hospital, 32 Ring 1 Road, Chengdu, Sichuan 610000, P.R. China

E-mail: hebin0321@yeah.net

${ }^{*}$ Contributed equally

Abbreviations: NSCLC, non-small cell lung cancer; CCK-8, Cell Counting Kit-8; PA-MSHA, pseudomonas aeruginosa-mannosesensitive haemagglutinin; HCC, hepatocellular carcinoma; MR, mannose receptor; CRC, colorectal cancer; EGFR-TK, epidermal growth factor receptor-tyrosine kinase

Key words: NSCLC, mannose, proliferation, cisplatin, apoptosis, metastasis

\section{Introduction}

In China, lung cancer is the leading cause of cancer death in males and the second leading cause of death in females (1). Lung cancer is also one of the leading causes of cancer-associated mortality globally (2). Lung cancer can be classified as small cell lung cancer and non-small cell lung cancer (NSCLC) (2). The standard first-line therapies for lung cancer include surgery, radiotherapy, chemotherapy, targeted therapy and immunotherapy (2); however, NSCLC is characterised by both aggressive behaviour and a poor response to chemotherapy (3). Moreover, secondary resistance often occurs as a result of exposure to chemotherapeutic agents (4). Therefore, it is important to explore new strategies to treat NSCLC.

Gonzalez et al (5), reported that mannose, a type of monosaccharide, induced growth retardation in various tumour types in vitro and enhanced cell death in response to major forms of chemotherapy in several cancers, including osteogenic sarcoma, ovarian cancer and pancreatic cancer. Whether mannose exerts antigrowth effects on NSCLC remains to be elucidated. The present study aimed to identify the effects of mannose on the proliferation, cell cycle progression, cisplatin-mediated apoptosis and metastasis of A549 and H1299 cells, in order to determine the mechanisms responsible for the mannose-induced anticancer effects.

\section{Materials and methods}

Cancer cell lines. A549 and H1299 lung adenocarcinoma cells were purchased from the American Type Culture Collection. 293FT cells were purchased from the Shanghai Cell Bank of the Chinese Academy of Sciences. Cells were cultured in Dulbecco's modified Eagle's medium (DMEM; Thermo Fisher Scientific, Inc.) supplemented with 10\% FBS (Biological Industries), streptomycin $(100 \mathrm{U} / \mathrm{ml})$ and penicillin $(100 \mathrm{U} / \mathrm{ml})$. All cells were incubated at $37^{\circ} \mathrm{C}$ in an incubator containing $5 \% \mathrm{CO}_{2}$. Mannose was dissolved in water, and the concentration of the storage stock was $500 \mathrm{mM}$ (stored at $-20^{\circ} \mathrm{C}$ ).

Cell Counting Kit-8(CCK-8) assay. Cell viability was detected with a CCK-8 assay (Dojindo Molecular Technologies, Inc.) according to the manufacturer's protocol. Cancer cells 
$(5,000$ cells/well $)$ were seeded in $96-$ well plates in complete culture medium for $24 \mathrm{~h}$. To evaluate the antitumour effects of mannose on A549 and H1299 cells in vitro, different concentrations $(0,15,30$ and $60 \mathrm{mM})$ of mannose were used to treat cancer cells for $24 \mathrm{~h}$ at $37^{\circ} \mathrm{C}$. The $\mathrm{IC}_{50}$ values of cisplatin against A549 and H1299 cells were 34.08 and $23.12 \mu \mathrm{M}$, respectively, which were calculated using GraphPad Prism v6 (GraphPad Software, Inc.). In addition, $30 \mathrm{mM}$ mannose was used to treat cancer cells for 12, 24 or $48 \mathrm{~h}$. To determine whether mannose could augment cisplatin-mediated apoptosis in lung cancer, $30 \mathrm{mM}$ mannose, $30 \mu \mathrm{M}$ cisplatin or mannose + cisplatin was used to treat cancer cells for $24 \mathrm{~h}$ at $37^{\circ} \mathrm{C}$. Then flow cytometry and western blotting were performed to evaluate the effects of mannose on cisplatin-mediated apoptosis of lung cancer cells.

Flow cytometry-based method for evaluating the cell cycle distribution. To assess the influence of mannose on the cell cycle progression of lung cancer cells, after being treated with $30 \mathrm{mM}$ mannose for $24 \mathrm{~h}$, cancer cells were harvested and fixed with $70 \%$ ethanol at $-20^{\circ} \mathrm{C}$ for $24 \mathrm{~h}$. Then, cells were stained with $0.1 \mathrm{mg} / \mathrm{ml}$ propidium iodide (PI; Sigma-Aldrich; Merck KGaA) for 15 min at room temperature in the dark, and the cell cycle distribution was evaluated and analysed using an Accuri C6 flow cytometer (BD Biosciences).

Flow cytometry-based method for evaluating apoptosis. To evaluate the effects of mannose on cisplatin-induced apoptosis, cell samples were grouped as follows: Control, $30 \mathrm{mM}$ mannose, $30 \mu \mathrm{M}$ cisplatin and $30 \mathrm{mM}$ mannose $+30 \mu \mathrm{M}$ cisplatin. After $24 \mathrm{~h}$, cells were collected for cell apoptosis analysis. Apoptotic cells were identified using flow cytometric analysis with an Annexin V-FITC kit (Invitrogen; Thermo Fisher Scientific, Inc.) according to the manufacturer's instructions. Samples were stained with annexin-V-FITC and PI for $15 \mathrm{~min}$ at room temperature in the dark. In the four fields on the original images, the dots indicate the number of Annexin V-/PI- (the bottom left field, indicating live cells), Annexin V+/PI- (the bottom right field, indicating early apoptotic cells), Annexin V+/PI+ (the top right field, indicating late apoptotic cells), and Annexin V-/PI+ (the top left field, indicating dead cells) cells. The percent of apoptotic cells was calculated as follows: Apoptosis rate $(\%)=$ rate of early apoptotic cells $(\%)+$ rate of late apoptotic cells $(\%)$. The apoptotic rate was evaluated and analysed using an Accuri C6 flow cytometer (BD Biosciences).

Transwell migration and invasion assays. To detect the invasive ability of cancer cells in vitro, $5 \mu 1$ of Matrigel (BD Biosciences) was spread in the upper chamber of a 24-well Transwell plate (BD Biosciences). After being treated with or without $30 \mathrm{mM}$ mannose for $24 \mathrm{~h}$, cancer cells were resuspended at a density of $1 \times 10^{5}$ cells $/ \mathrm{ml}$ in DMEM without FBS Then, $0.5 \mathrm{ml}$ of the cell suspension was added to each upper chamber of the 24-well plate, and $0.5 \mathrm{ml}$ of DMEM containing $20 \%$ FBS to the lower chambers. After $24 \mathrm{~h}$, the Matrigel and NSCLC cells in the upper chambers were cleaned carefully and thoroughly. The upper and lower chambers were washed once with $1 \mathrm{X}$ PBS, and cells were fixed with $4 \%$ paraformaldehyde for $20 \mathrm{~min}$ at room temperature. NSCLC cells on the lower surface of the upper chambers were stained with $0.25 \%$ crystal violet, which was dissolved in $20 \%$ methanol, for
$30 \mathrm{~min}$ at room temperature. The samples were washed twice with PBS, and the membranes of the chambers were sealed with cover glue (Leica Microsystems GmbH). Membranes were wetted with PBS and observed under a microscope (Nikon Corporation); 5 random fields (magnification, x20) were selected, the NSCLC cells were counted, and the average numbers were analysed and compared.

For detection of the migration ability of cancer cells in vitro, the protocol was the same as that used for the invasion assays, but Matrigel was not used.

Western blot assay. For western blotting, cancer cells were treated with 30 and $60 \mathrm{mM}$ mannose for $24 \mathrm{~h}$. Then, total protein lysate from cancer cells was extracted with RIPA lysis buffer (Shanghai Yeasen Biotechnology Co.) and the protein concentration was evaluated by bicinchoninic acid assay (Thermo Fisher Scientific, Inc.). A total of 10-20 $\mu \mathrm{g}$ protein lysate per lane was separated by $10 \%$ SDS-PAGE and transferred to polyvinylidene difluoride membranes (EMD Millipore). Membranes were blocked with 5\% non-fat milk in Tris- $\mathrm{HCl}$ buffer solution containing $0.1 \%$ Tween 20 (TBST) for $1 \mathrm{~h}$ at room temperature and incubated separately with primary antibodies against the following proteins at $4^{\circ} \mathrm{C}$ overnight: p21 (1:1,000; cat. no. ab218311; Abcam), proliferating cell nuclear antigen (PCNA; 1:1,000; cat. no. ab92552; Abcam), Bcl-2 (1:1,000; cat. no. ab185002; Abcam), Bax (1:1,000; cat. no. ab32503; Abcam), cleaved caspase-3 (1:1,000; cat. no. ab2302; Abcam), GAPDH (1:10,000; cat. no. ab181602; Abcam), matrix metalloproteinase 2 (MMP2; 1:1,000; cat. no. ab97779; Abcam), E-cadherin (1:1,000; cat. no. ab1416; Abcam), N-cadherin (1:1,000; cat. no. ab18203; Abcam), phosphorylated (p)-AKT (Ser473; 1:1,000; cat. no. ab81283; Abcam), total AKT (1:1,000; cat. no. ab8805; Abcam), p-ERK1/2 (1:1,000; cat. no. ab223500; Abcam), and total ERK (1:1,000; cat. no. ab17942; Abcam). After washing with TBST, membranes were incubated with horseradish peroxidase-conjugated anti-rabbit $\operatorname{IgG}(1: 2,500$; cat. nos. ab6721 and ab6728; Abcam) for $1 \mathrm{~h}$ at room temperature. Visualization of blots was performed using a standard protocol for electro-chemiluminescence (cat. no. ab133406; Abcam). The levels of GAPDH were used as the internal standards. Densitometry was performed using Image J software (v1.47; National Institutes of Health).

Statistical analysis. Data are presented as the mean \pm SD. Student's t-test or one-way analysis of variance (ANOVA) was used to evaluate statistical differences via GraphPad Prism v6 (GraphPad Software, Inc.). Tukey's multiple comparisons test was used to analyze data following ANOVA. $\mathrm{P}<0.05$ was considered to indicate a statistically significant difference.

\section{Results}

Mannose inhibits the viability of lung cancer cells in vitro. Mannose is a carbohydrate; the structure of mannose is shown in Fig. 1A. A549, H1299 and 293FT cells were used in the present study to evaluate the antigrowth effects of mannose on NSCLC and non-cancer cell lines in vitro. A CCK-8 assay showed that mannose does not significantly affect the viability of 293FT cells ( $n=3$; Fig. 1B). Conversely, mannose 
A<smiles>O=C[C@H](O)[C@H](O)[C@H](O)[C@H](O)CO</smiles>

C

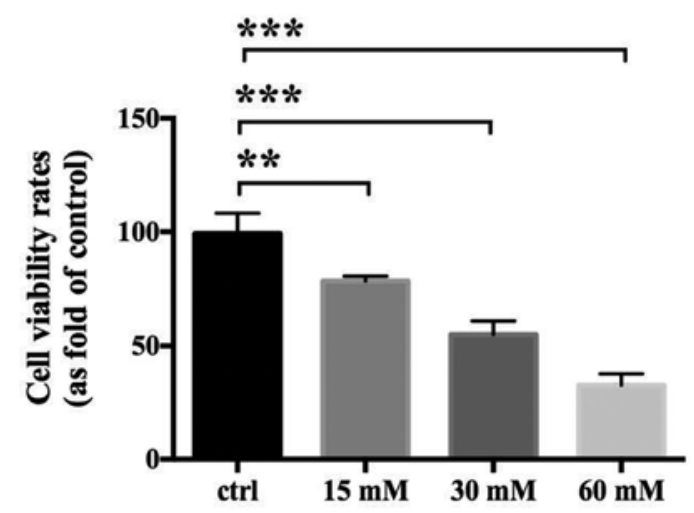

E

A549

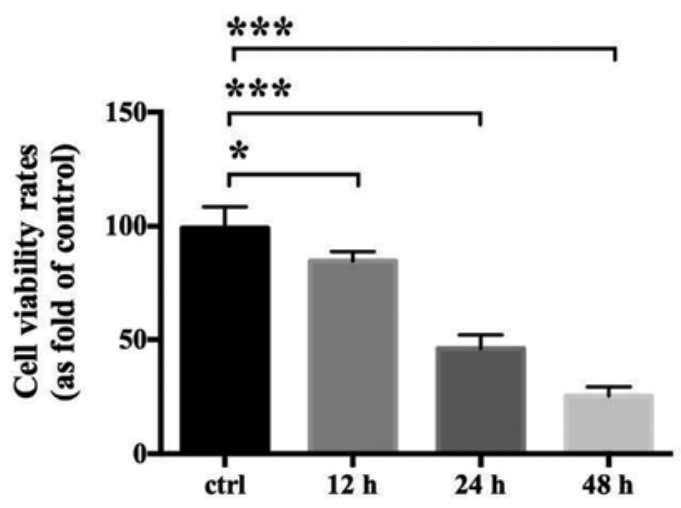

B

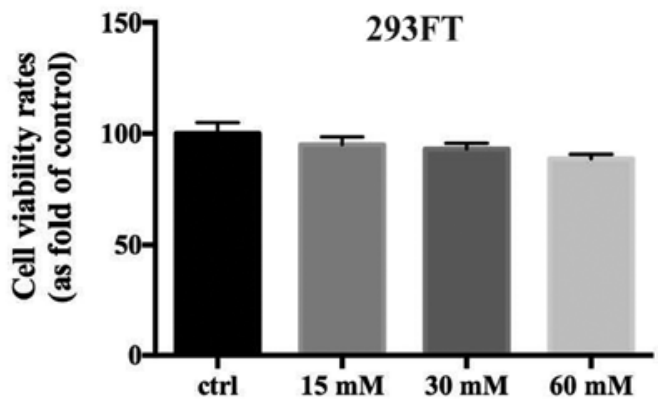

D

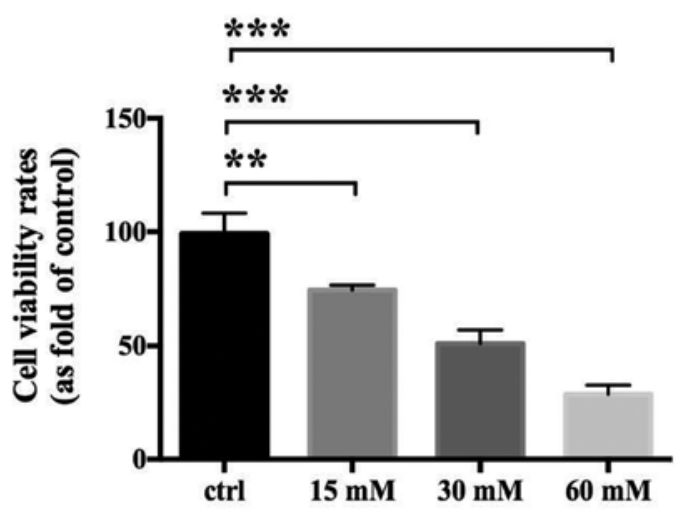

F

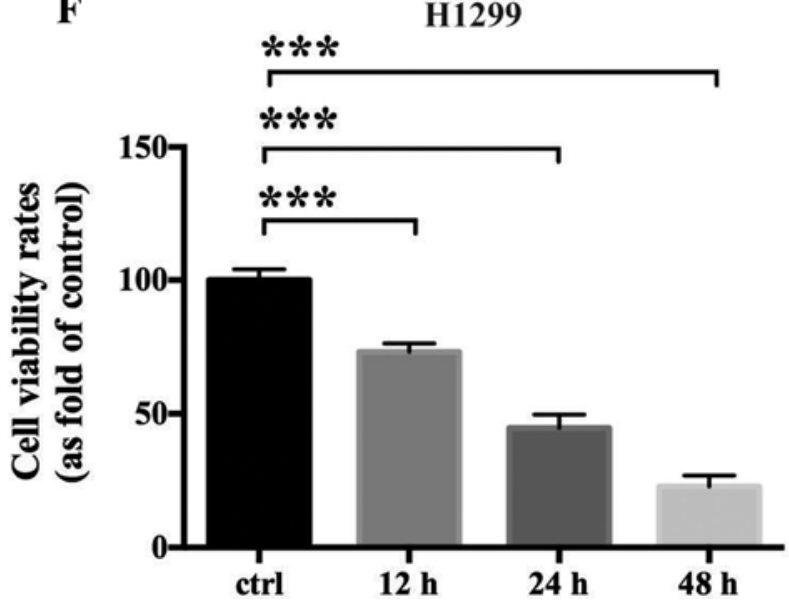

Figure 1. Mannose inhibits the proliferative ability of NSCLC cells in vitro. (A) Structure of mannose. (B) CCK-8 cell viability assays revealed that mannose exerted non-significant antigrowth effects on the non-cancer cell line 293FT in vitro. CK-8 assay results showed that mannose exerted anticancer effects on NSCLC in vitro in both $(\mathrm{C}$ and $\mathrm{D})$ dose- and $(\mathrm{E}$ and $\mathrm{F})$ time-dependent manners. Data are presented as the mean \pm standard deviation. ${ }^{*} \mathrm{P}<0.05,{ }^{* *} \mathrm{P}<0.01$, ${ }^{* * *} \mathrm{P}<0.001$. NSCLC, non-small cell lung cancer; ctrl, control; CCK-8, Cell Counting Kit-8.

significantly inhibited the viability of A549 and H1299 cells dose-dependently at $24 \mathrm{~h}$ in vitro ( $\mathrm{n}=3$; Fig. $1 \mathrm{C}$ and D). The $\mathrm{IC}_{50}$ of mannose against A549 and $\mathrm{H} 1299$ cells at $24 \mathrm{~h}$ was $\sim 30 \mathrm{mM}$, which was used as the experimental concentration in subsequent assays. In addition, $30 \mathrm{mM}$ mannose significantly reduced the viability of A549 and H1299 cells in a time-dependent manner in vitro $(n=3$; Fig. $1 \mathrm{E}$ and $\mathrm{F})$.

Mannose induces G0/G1 phase arrest in lung cancer cells in vitro. To further examine whether mannose inhibits the proliferation of NSCLC cells by regulating cell cycle progression, a flow cytometry-based method was used. The results showed that after treatment with $30 \mathrm{mM}$ mannose for $24 \mathrm{~h}$, the cell cycle distribution of A549 and H1299 cells was significantly altered compared with the control group (Fig. 2A-D). It was revealed that mannose increased the population of $\mathrm{G} 0 / \mathrm{G} 1$-phase and reduced the population of S/G2/M-phase A549 (n=3; Fig. 2A and B) and H1299 cells in vitro $(\mathrm{n}=3$; Fig. $2 \mathrm{C}$ and $\mathrm{D})$. Next, the effects of mannose on the expression levels of two regulators of the cell cycle, PCNA and p21, were further investigated via western blotting. The western blot assay results showed that treatment with 30 or $60 \mathrm{mM}$ mannose for $24 \mathrm{~h}$ downregulated PCNA expression in both A549 and H1299 cells in vitro; in contrast, 
A

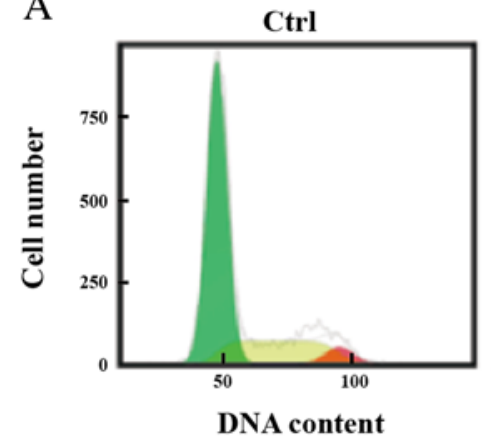

C

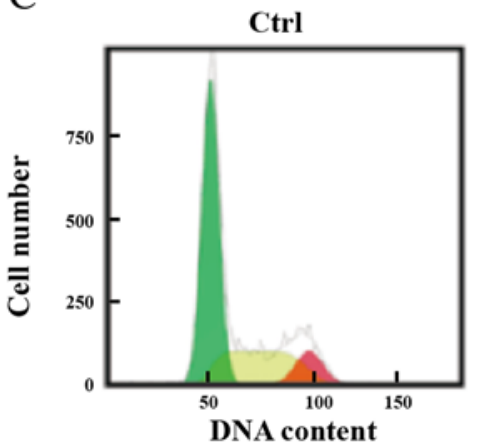

Mannose

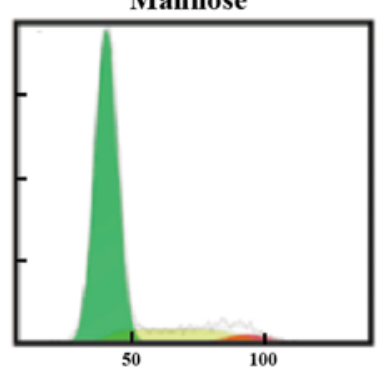

DNA content

Mannose

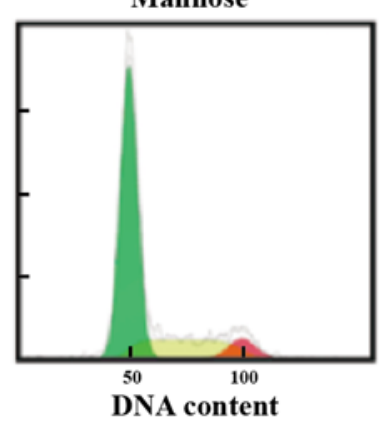

A549
B

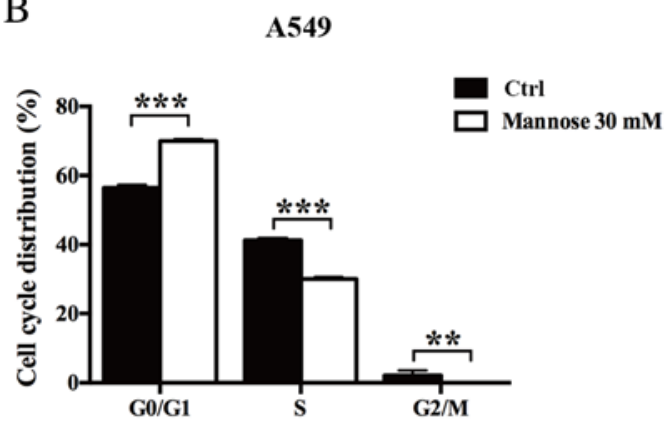

$\mathrm{D}$

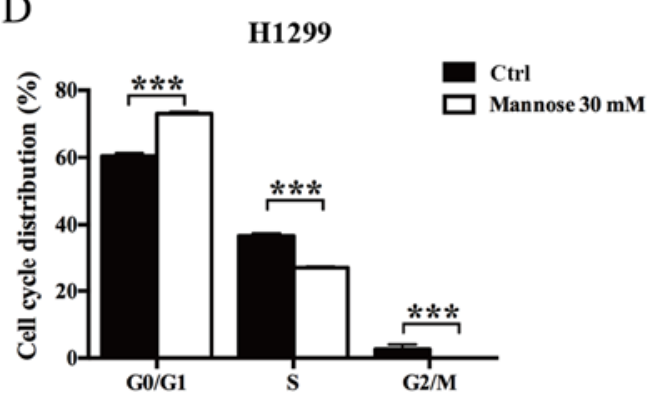

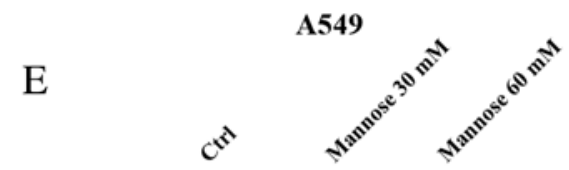

PCNA

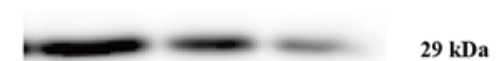

p21

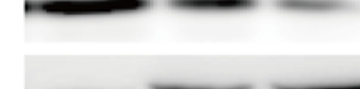

$29 \mathrm{kDa}$

GAPDH

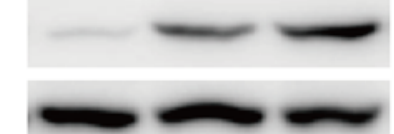

$21 \mathrm{kDa}$

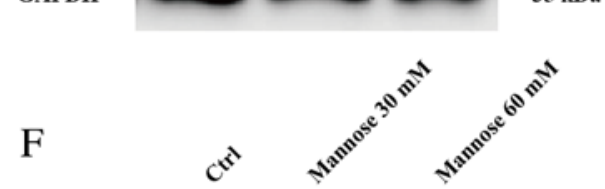

$5 \mathrm{kDa}$
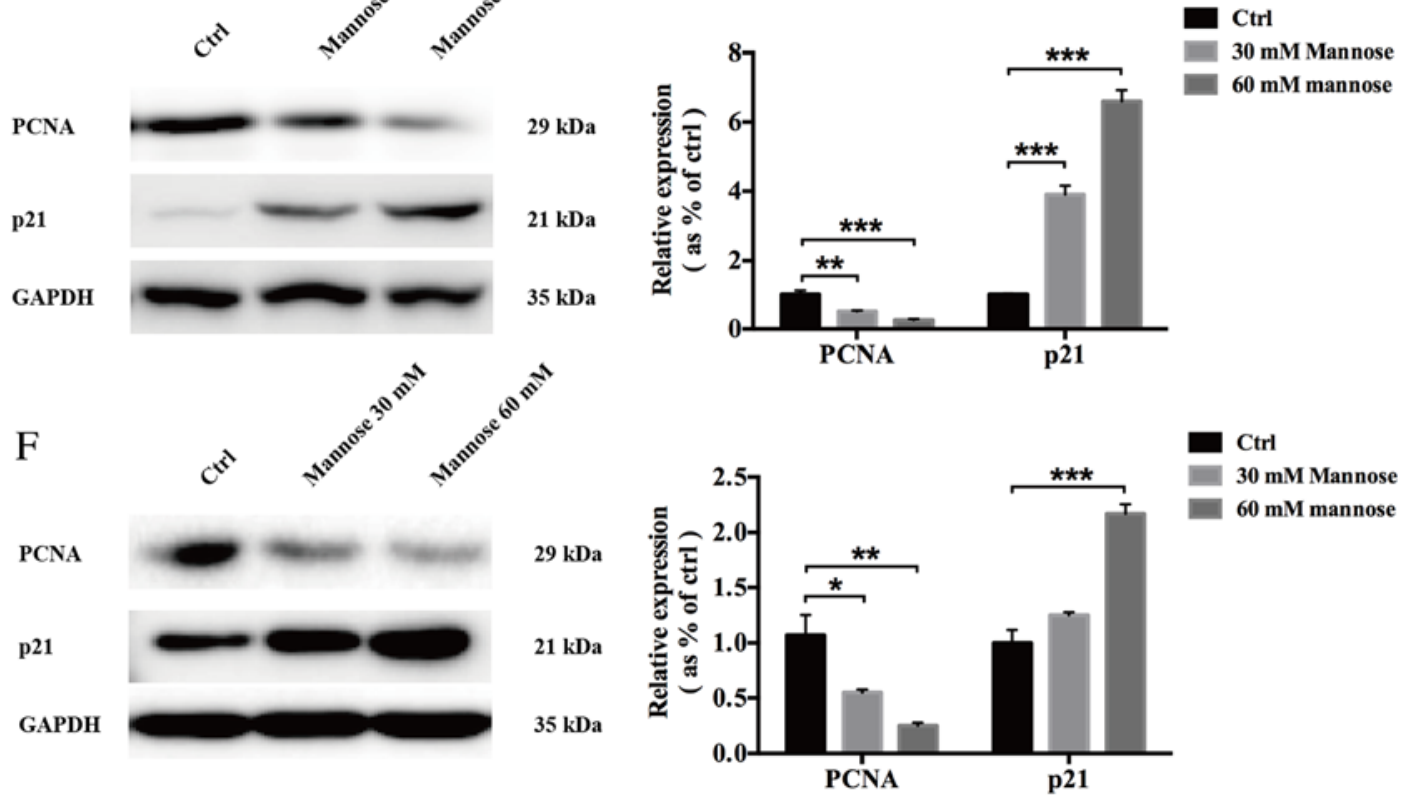

Figure 2. Mannose induces G0/G1 phase arrest in NSCLC cells in vitro. (A-D) Flow cytometry was performed to assess the effects of 30 mM mannose on the cell cycle distribution of NSCLC cells in vitro. (E and F) Western blotting was conducted to evaluate the effects of different concentrations of mannose $(0,30$ and $60 \mathrm{mM}$ ) on the expression of PCNA and p21, regulators of cell cycle progression. Data are presented as the mean \pm standard deviation. ${ }^{*} \mathrm{P}<0.05,{ }^{* *} \mathrm{P}<0.01$, ${ }^{* * *} \mathrm{P}<0.001$. One-way ANOVA was used in parts (A) and (B). NSCLC, non-small cell lung cancer; ctrl, control; PCNA, proliferating cell nuclear antigen.

mannose treatment significantly upregulated p21 expression in NSCLC cells (Fig. 2E and F). Furthermore, the effects of mannose on the expression levels of PCNA and p21 increased in a concentration-dependent manner. Mannose may induce G0/G1 cell cycle arrest in A549 and H1299 cells by affecting the expression of PCNA and $\mathrm{p} 21$.

Mannose increases cisplatin-mediated apoptosis of lung cancer cells in vitro. Cisplatin resistance in NSCLC is an urgent problem that requires overcoming to improve the outcomes of lung cancer therapy (6). In the present study, flow cytometric and western blot analyses were conducted to evaluate whether mannose could induce apoptosis in A549 and H1299 cells in vitro, and whether it could augment cisplatin-mediated cellular death. First, a CCK-8 cell viability assay was used to determine the $\mathrm{IC}_{50}$ of cisplatin against both A549 and H1299 cells. The $\mathrm{IC}_{50}$ values of cisplatin against A549 and H1299 cells were $34.08 \mu \mathrm{M}$ and $23.12 \mu \mathrm{M}$, respectively (Fig. 3A), so $30 \mu \mathrm{M}$ 
$\mathbf{A}$

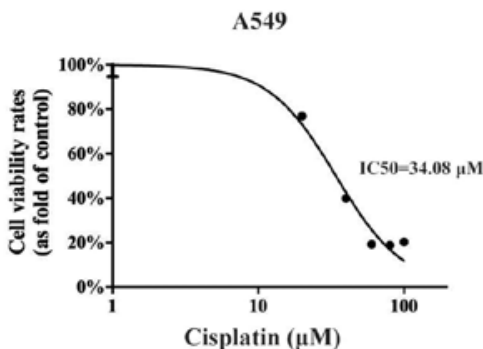

B A549
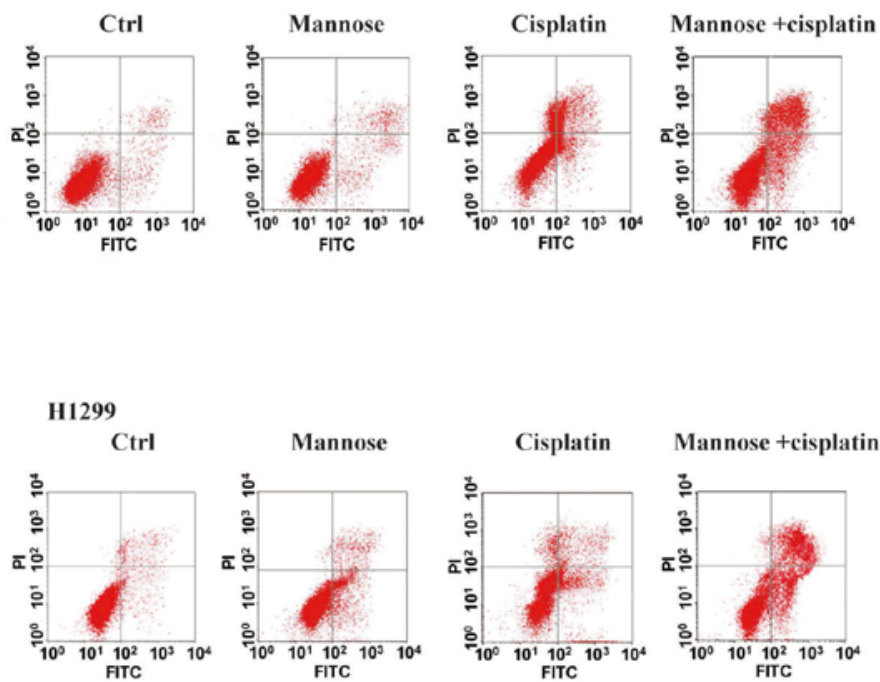

H1299
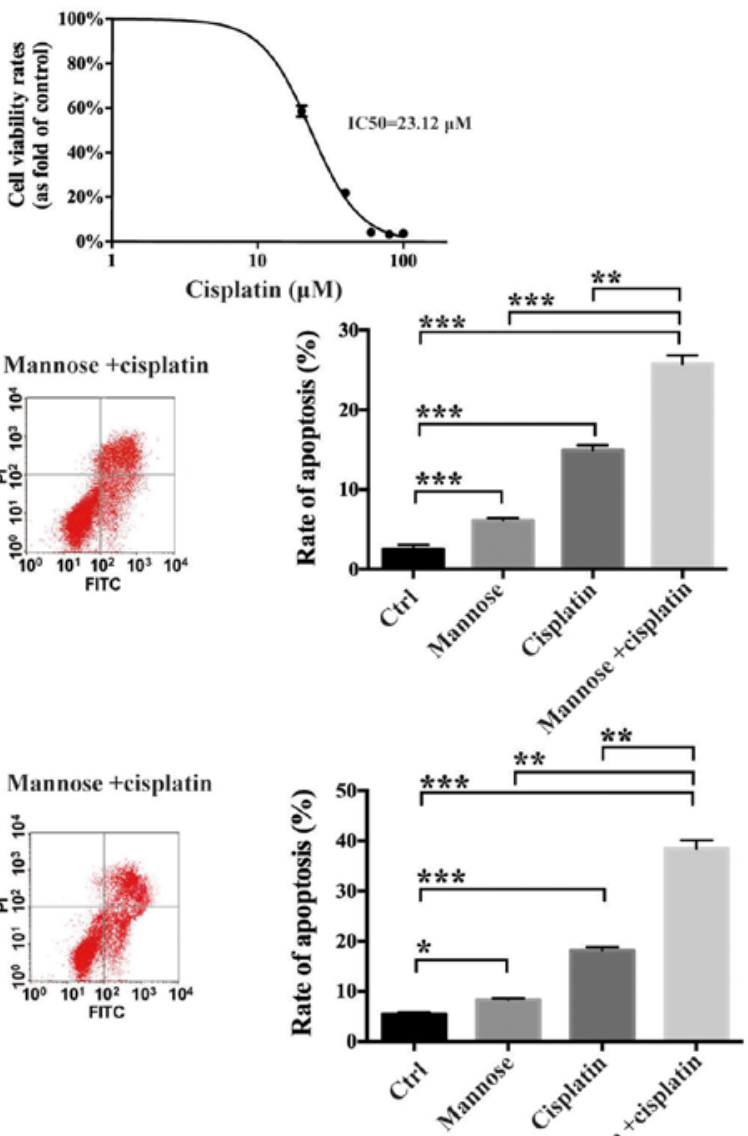

C
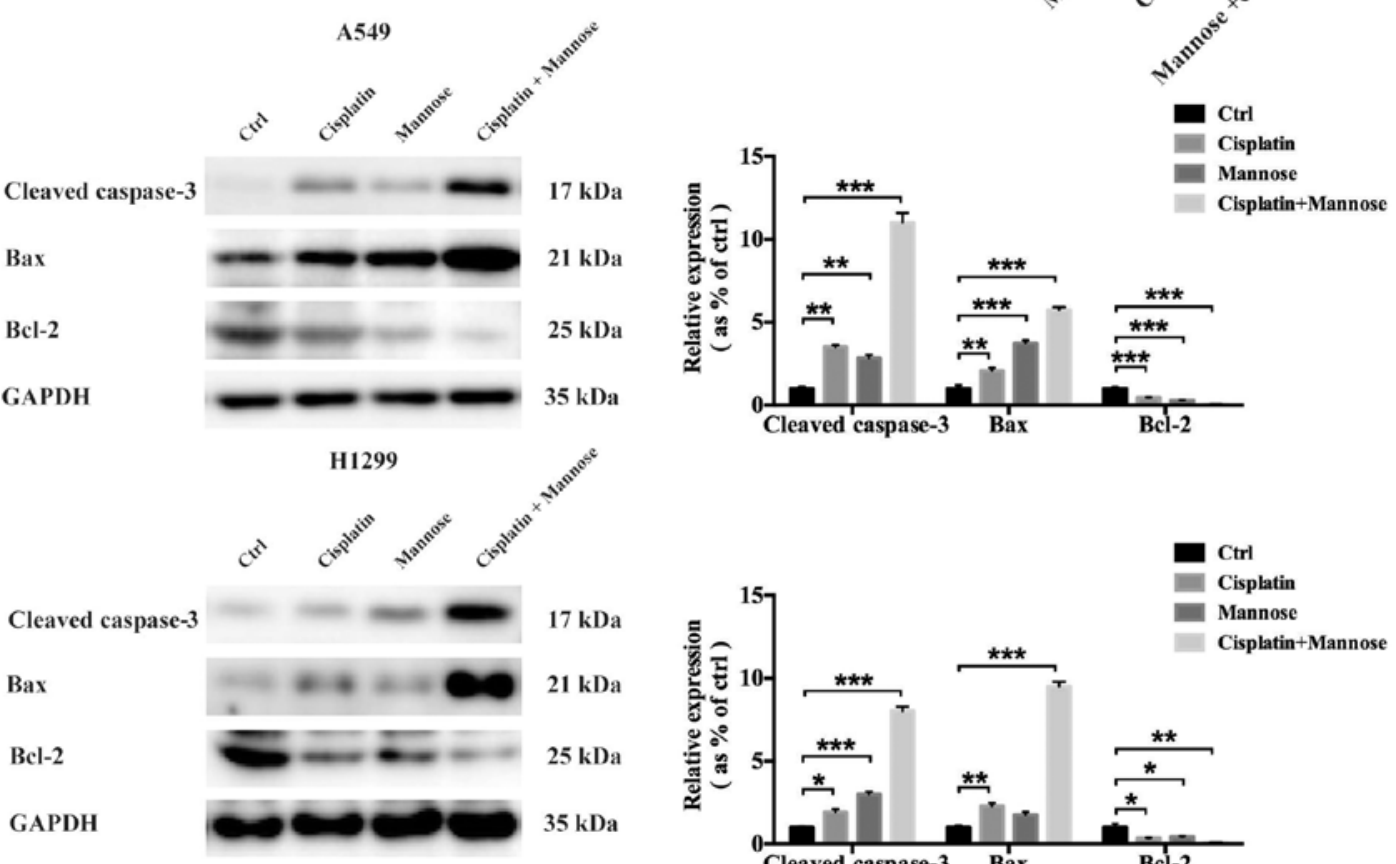

\section{D}


A

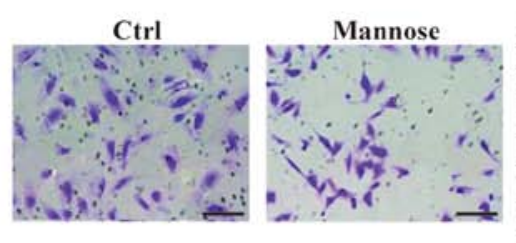

A549

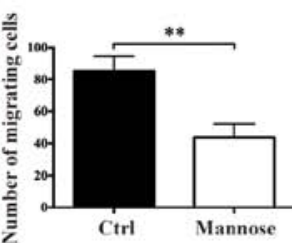

Ctrl Mannose
H1299
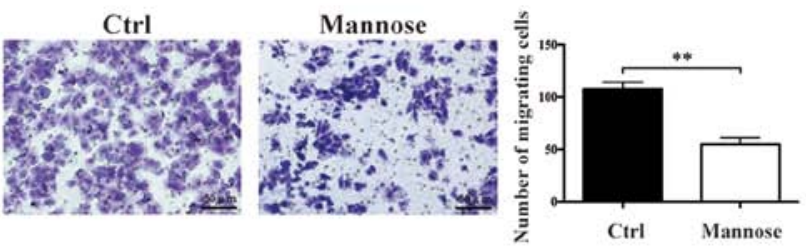

B

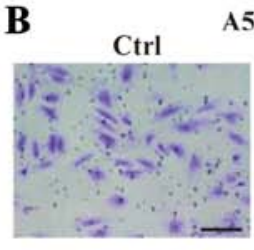

A549
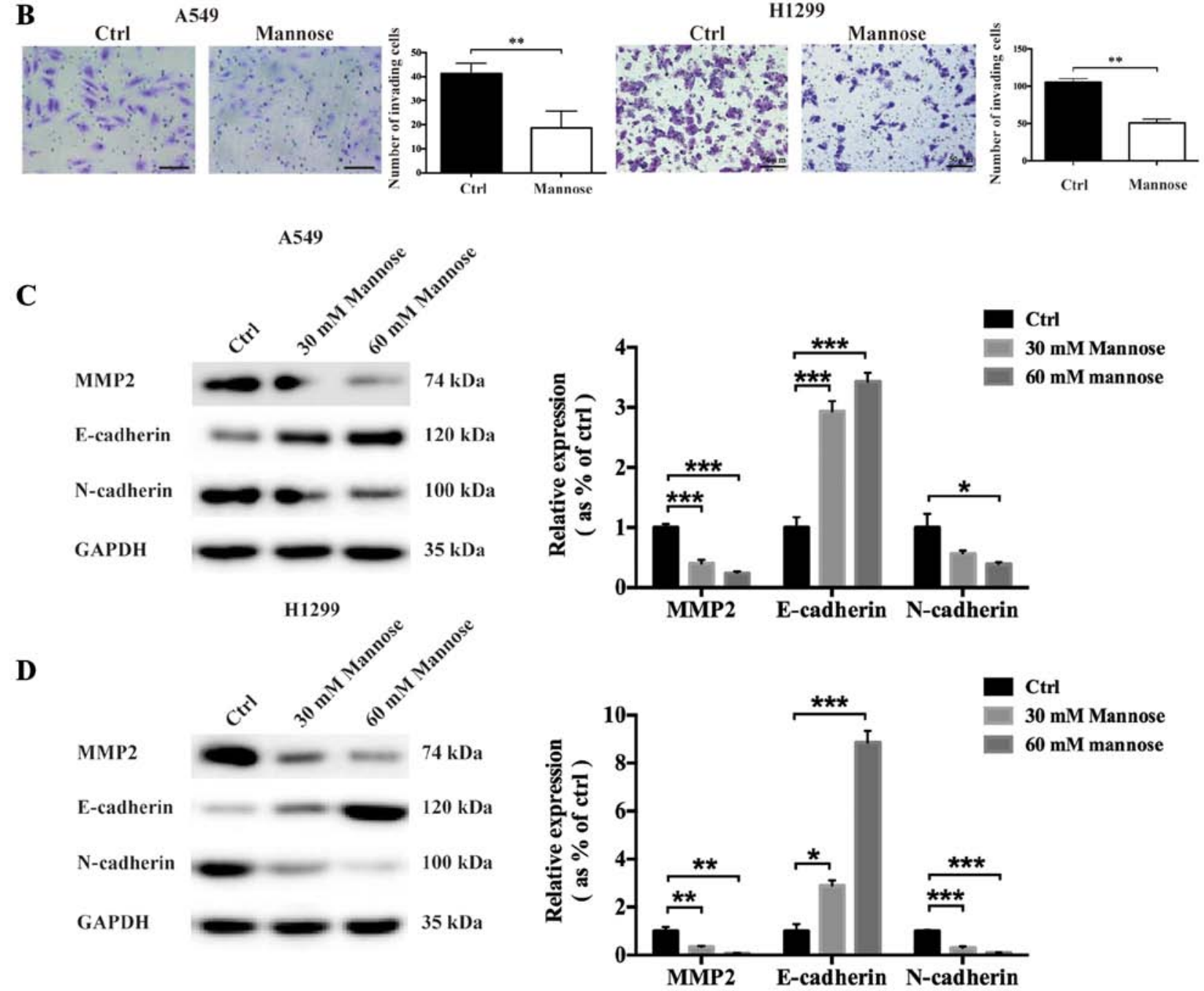

Figure 4. Mannose reduces the migratory and invasive abilities of lung cancer cells in vitro. (A) Transwell migration assays were used to evaluate the effects of mannose on the migratory abilities of A549 and H1299 cells (scale bar =50 $\mu \mathrm{m}$ ). (B) Transwell invasion assays were used to evaluate the effects of mannose on the invasive abilities of A549 and $\mathrm{H} 1299$ cells (scale bar $=50 \mu \mathrm{m})$. (C and D) Western blotting was conducted to evaluate the effects of different concentrations of mannose $(0,30$ and $60 \mathrm{mM})$ on the expression of epithelial-mesenchymal transition-associated proteins, including MMP2, E-cadherin and N-cadherin. Data are presented as the mean \pm standard deviation. ${ }^{*} \mathrm{P}<0.05,{ }^{* *} \mathrm{P}<0.01,{ }^{* * * *} \mathrm{P}<0.001$. MMP2, matrix metalloproteinase 2; ctrl, control.

Furthermore, under treatment with the combination of $30 \mathrm{mM}$ mannose $+30 \mu \mathrm{M}$ cisplatin, the percentage of apoptotic cancer cells was further increased compared with single-agent mannose or cisplatin treatment (Fig. 3B). To further explore the molecular changes responsible for this phenomenon, western blotting was conducted to measure the expression levels of apoptosis-related proteins, including cleaved caspase-3, Bax and Bcl-2. The results showed that treatment with $30 \mathrm{mM}$ mannose or $30 \mu \mathrm{M}$ cisplatin for $24 \mathrm{~h}$ downregulated Bcl-2 expression in both A549 and H1299 cells in vitro. In contrast, the expression of both cleaved caspase- 3 and Bax in NSCLC cells was upregulated (Fig. 3C and E). Furthermore, mannose significantly augmented the effects of cisplatin on the expression of cleaved caspase-3, Bax and Bcl-2. Mannose may thus enhance the cisplatin-mediated apoptosis of A549 and H1299 cells by affecting the expression of $\mathrm{Bax} / \mathrm{Bcl}-2$ and caspase- 3 .

Mannose reduces the migration and invasive abilities of lung cancer cells in vitro. The metastasis of malignant tumours is one of the primary complications of cancers, including NSCLC (7). To determine whether mannose could inhibit the invasive abilities of NSCLC cells, Transwell migration and invasion, and western blot assays were used to evaluate the anti-invasion effects of mannose. Following treatment with 30 
A

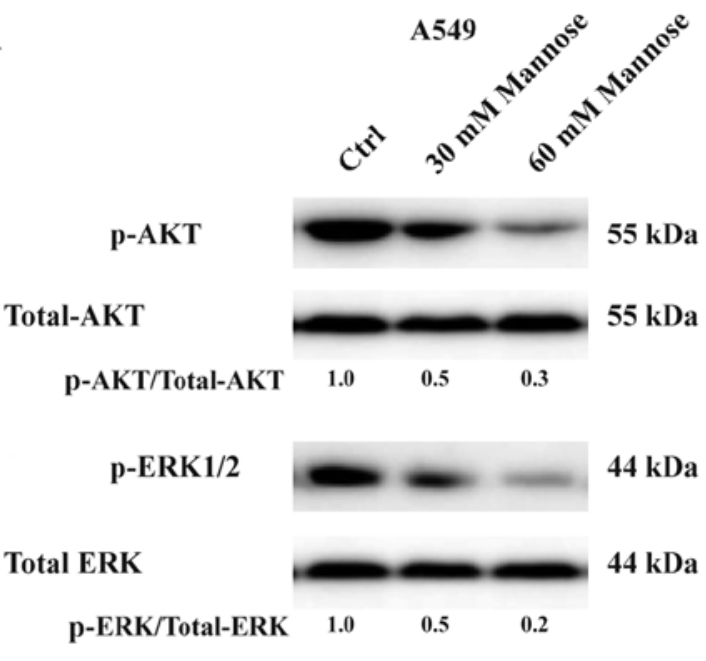

GAPDH

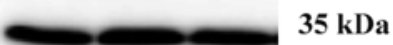

B

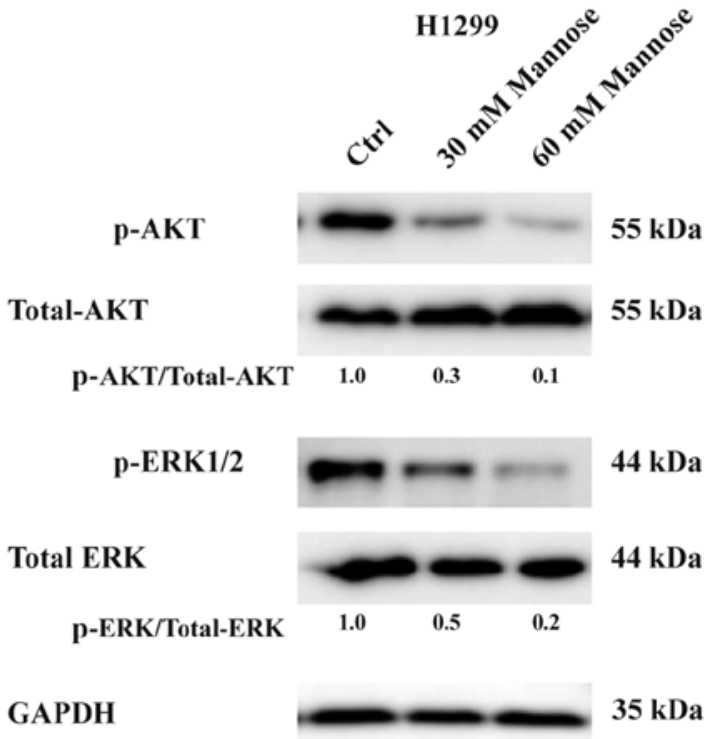

$35 \mathrm{kDa}$
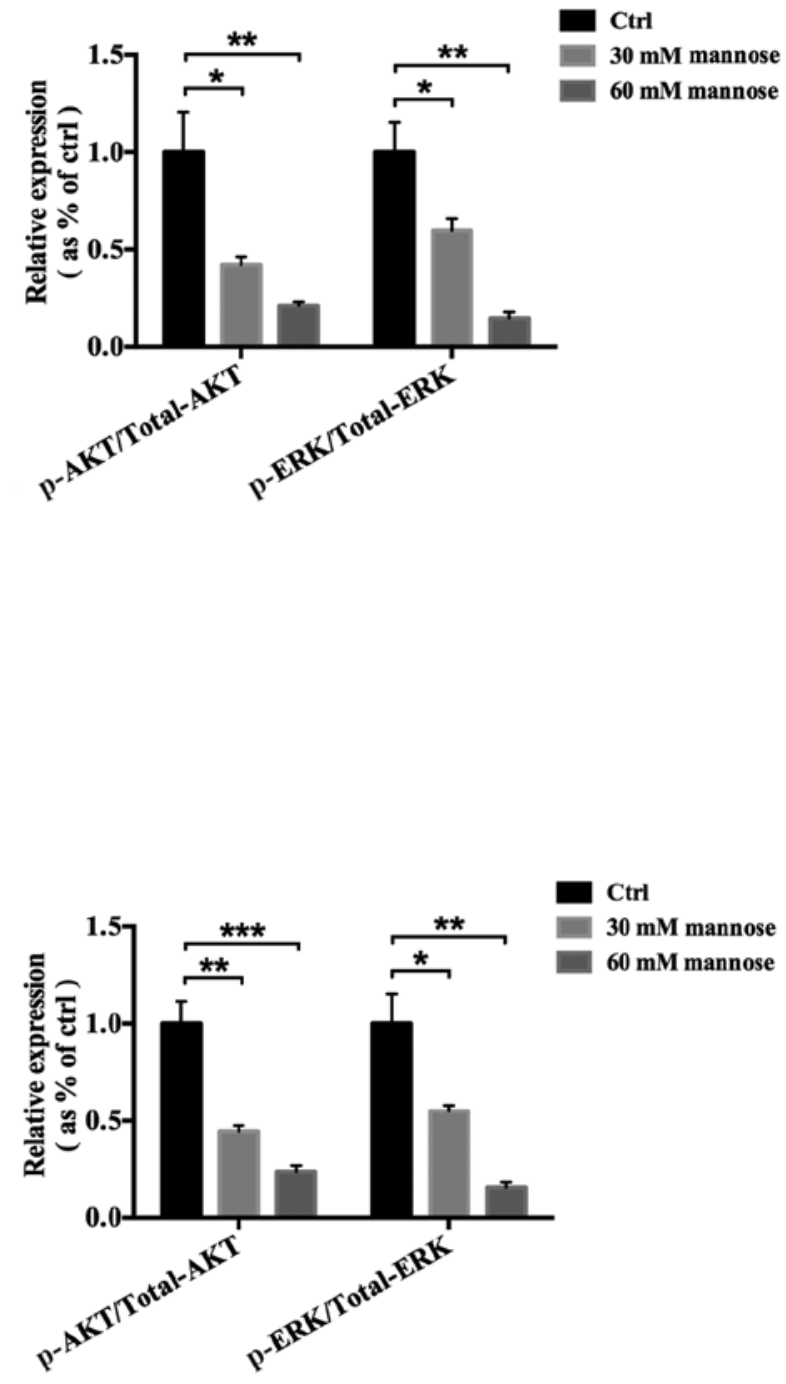

Figure 5. Mannose inhibits the PI3K/AKT and ERK signalling pathways in non-small cell lung cancer. (A and B) Western blotting was conducted to evaluate the effects of different concentrations of mannose $(0,30$ and $60 \mathrm{mM})$ on the activation of the PI3K/AKT and ERK signalling pathways. ${ }^{*} \mathrm{P}<0.05,{ }^{* *} \mathrm{P}<0.01$, ${ }_{* * * *} \mathrm{P}<0.001$. Ctrl, control; p, phosphorylated.

mM mannose for $24 \mathrm{~h}$, the migration of A549 and H1299 cells was significantly reduced in vitro (Fig. 4A). The Transwell invasion assays showed that mannose also reduced the invasive abilities of NSCLC cells in vitro (Fig. 4B). To further explore the effects of mannose on epithelial-mesenchymal transition (EMT) in A549 and H1299 cells, western blotting was used to assess the expression of MMP2, E-cadherin and $\mathrm{N}$-cadherin. It was observed that treatment with 30 or $60 \mathrm{mM}$ mannose for $24 \mathrm{~h}$ downregulated $\mathrm{N}$-cadherin and MMP2 expression in both A549 and H1299 cells in vitro (Fig. 4C and D). In contrast, mannose treatment significantly upregulated E-cadherin expression in NSCLC cells. Furthermore, the effects of mannose on the expression of MMP2, E-cadherin and N-cadherin significantly increased in a concentration-dependent manner. Mannose may inhibit the metastatic ability of NSCLC cells by inhibiting EMT.
Mannose inhibits the PI3K/AKT and ERK signalling pathways in lung cancer. A number of studies have reported the contribution of the PI3K/AKT signalling pathway (8) and mitogen-activated protein kinase (MAPK/ERK) signalling pathway $(9,10)$ to the progression of lung cancer, including the promotion of proliferation, reductions in apoptosis and the facilitation of metastasis. In the current study, western blotting was conducted to examine the effects of mannose on the activation of the PI3K/AKT and ERK signalling pathways in A549 and H1299 cells in vitro, including the levels of p-AKT (Ser473) and p-ERK1/2. It was found that treatment with 30 or $60 \mathrm{mM}$ mannose for $24 \mathrm{~h}$ decreased the levels of p-AKT (Ser473) and p-ERK1/2 in both A549 and H1299 cells in vitro (Fig. $5 \mathrm{~A}$ and $\mathrm{B}$ ). The ratios of $\mathrm{p}-\mathrm{AKT} / \mathrm{total} \mathrm{AKT}$ and p-ERK1/2/total ERK were significantly decreased in A549 and H1299 cells. The inhibitory regulation of the AKT and 
ERK signalling pathways by mannose increased in a concentration-dependent manner. Mannose may exert anticancer effects on A549 and H1299 cells by inhibiting the PI3K/AKT and ERK signalling pathways.

\section{Discussion}

The present study identified that mannose exerted anticancer effects on NSCLC cell lines (A549 and H1299) in vitro by inhibiting proliferation, inducing G0/G1 cell cycle arrest, promoting cisplatin-induced apoptosis and decreasing invasion. Li et al (11), observed that Pseudomonas aeruginosa mannose-sensitive haemagglutinin (PA-MSHA) exhibited antitumour properties against hepatocellular carcinoma (HCC) through mannose-binding activity and downregulation of the epidermal growth factor receptor (EGFR)/Akt/I $\mathrm{B} \beta / \mathrm{NF}-\kappa \mathrm{B}$ pathway. PA-MSHA induced significant cell proliferation inhibition and cell cycle arrest by decreasing the expression levels of PCNA, cyclin-D1/E, cyclin-dependent kinases 2/4 and increasing the levels of p21 and p27 (11). PA-MSHA also suppressed the migration, invasion and adhesion of HCC cells by inhibiting EMT, consistent with the present study (11). Sturge et al (12), reported that the mannose receptor (MR) was important for macrophage migration; bone marrow-derived macrophages obtained from MR-deficient mice exhibited an increase in random cell migration. Ding et al (13), hypothesised that MR could be a novel biomarker for colorectal cancer (CRC), reporting that the expression of MR in CRC tissues was significantly upregulated compared with in paracancerous tissues. Furthermore, high expression of MR in CRC tissues was also associated with reduced overall survival and an increased degree of lymphatic metastasis. These findings highlight the potential value of exploring the effects of mannose and its receptor on cancer metastasis.

It has been reported that the PI3K-AKT and ERK1/2 signalling pathways, and the crosstalk between them, can facilitate cancer progression (14). Aberrant activation of the PI3K-AKT and ERK signalling pathways contributes to cancer growth, metastasis (15) and chemoresistance $(16,17)$. Furthermore, overactivation of the PI3K/AKT/mTOR pathway is one of the mechanisms of acquired resistance to EGFR-tyrosine kinase (EGFR-TK) inhibitors in patients with NSCLC carrying EGFR-activating mutations (18). The treatment of NSCLC has been modified due to emerging novel strategies against certain molecular targets, including PI3K (19), AKT and MAPK/ERK kinase (MEK) (20), mTOR (21) and ERK (22). The present study identified that mannose may decrease the activation of both AKT and ERK1/2, potentially contributing to its anticancer effects on NSCLC cells in vitro. These data indicated that mannose could be combined with AKT or ERK-targeted therapies in lung cancer. However, additional experiments should be conducted to explore the molecular mechanisms underlying the anticancer effects of mannose against NSCLC in vitro and in vivo.

Abnormal metabolism is involved in various malignant behaviours of NSCLC, including cell survival, metastatic potential and disease progression (23). Mannose in animal cells is phosphorylated by hexokinase and subsequently isomerised by mannose phosphate isomerase (MPI) to fructose-6-P, which is used in the glycolysis pathway (24). de la Fuente and Hernanz (24), reported a significant decrease in MPI activity in splenic lymphoid cells from a leukaemia-bearing mouse model, which enhanced the toxicity of mannose towards splenic lymphoid cells. These authors also observed that MPI activity was downregulated in peripheral blood lymphocytes from patients with chronic lymphocytic leukaemia compared with in lymphocytes from healthy donors. The present study identified that mannose augmented the cisplatin-induced apoptosis of NSCLC cells in vitro, potentially via the $\mathrm{Bcl}-2 / \mathrm{Bax} /$ caspase- 3 pathway. However, the association between MPI and NSCLC is yet to be reported, to the best of our knowledge. In addition, whether MPI contributes to the chemoresistance of NSCLC remains unclear. Cazet et al (25), reported that MPI was a potential enzymatic target for glioma therapy; MPI knockdown significantly reduced glioma survival and increased radiosensitivity, indicating the potential value of combining mannose with MPI-targeted therapy for cancer treatment.

In summary, the present study revealed the anticancer activity of mannose against NSCLC cells in vitro via the inhibition of the AKT/ERK signalling pathway. Further experiments are required to explore the mechanisms underlying the anticancer properties of mannose, including its effects on mice bearing tumours. The present study provides a rationale for the potential application of mannose-based strategies against NSCLC.

\section{Acknowledgements}

Not applicable.

\section{Funding}

The study was funded by Science and Technology Research Program of Sichuan (grant no. 2016FX0092).

\section{Availability of data and materials}

The datasets and supporting materials generated during and/or analysed during the present study are available from the corresponding author on reasonable request.

\section{Authors' contributions}

YW participated in the design of the study and drafted the manuscript. SX collected and analyzed the data. BH designed the study, revised the manuscript and is responsible for authenticity of data. All authors read and approved the final manuscript.

\section{Ethics approval and consent to participate}

Not applicable.

\section{Patient consent for publication}

Not applicable.

\section{Competing interests}

The authors declare that they have no competing interests. 


\section{References}

1. Hong QY, Wu GM, Qian GS, Hu CP, Zhou JY, Chen LA, Li WM, Li SY, Wang K, Wang Q, et al; Lung Cancer Group of Chinese Thoracic Society; Chinese Alliance Against Lung Cancer: Prevention and management of lung cancer in China. Cancer 121 (Suppl 17): 3080-3088, 2015.

2. Torre LA, Siegel RL and Jemal A: Lung Cancer Statistics. Adv Exp Med Biol 893: 1-19, 2016.

3. Zhao Y and Adjei AA: New strategies to develop new medications for lung cancer and metastasis. Cancer Metastasis Rev 34 265-275, 2015.

4. Chang A: Chemotherapy, chemoresistance and the changing treatment landscape for NSCLC. Lung Cancer 71: 3-10, 2011.

5. Gonzalez PS, O'Prey J, Cardaci S, Barthet VJA, Sakamaki JI, Beaumatin F, Roseweir A, Gay DM, Mackay G, Malviya G, et al: Mannose impairs tumour growth and enhances chemotherapy. Nature 563: 719-723, 2018.

6. Hu C, Zhang M, Moses N, Hu CL, Polin L, Chen W, Jang H, Heyza J, Malysa A, Caruso JA, et al: The USP10-HDAC6 axis confers cisplatin resistance in non-small cell lung cancer lacking wild-type p53. Cell Death Dis 11: 328, 2020.

7. Nevel KS, DiStefano N, Lin X, Skakodub A, Ogilvie SQ, Reiner AS, Pentsova E and Boire A: A retrospective, quantitative assessment of disease burden in patients with leptomeningeal metastases from non-small-cell lung cancer. Neuro Oncol 22: 675-683, 2019.

8. Fu QF, Liu Y, Fan Y, Hua SN, Qu HY, Dong SW, Li RL, Zhao MY, Zhen Y, Yu XL, et al: Alpha-enolase promotes cell glycolysis, growth, migration, and invasion in non-small cell lung cancer through FAK-mediated PI3K/AKT pathway. J Hematol Oncol 8 $22,2015$.

9. Fong Y, Wu CY, Chang KF, Chen BH, Chou WJ, Tseng $\mathrm{CH}$, Chen YC, Wang HD, Chen YL and Chiu CC: Dual roles of extracellular signal-regulated kinase (ERK) in quinoline compound BPIQ-induced apoptosis and anti-migration of human non-small cell lung cancer cells. Cancer Cell Int 17: 37, 2017.

10. Wang H, Yu Z, Huo S, Chen Z, Ou Z, Mai J, Ding S and Zhang J: Overexpression of ELF3 facilitates cell growth and metastasis through PI3K/Akt and ERK signaling pathways in non-small cell lung cancer. Int J Biochem Cell Biol 94: 98-106, 2018.

11. Li T, Dong ZR, Guo ZY, Wang CH, Zhi XT, Zhou JW, Li DK, Chen ZT, Chen ZQ and Hu SY: Mannose-mediated inhibitory effects of PA-MSHA on invasion and metastasis of hepatocellular carcinoma

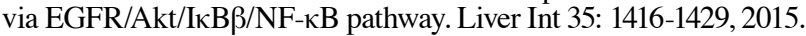

12. Sturge J, Todd SK, Kogianni G, McCarthy A and Isacke CM: Mannose receptor regulation of macrophage cell migration. J Leukoc Biol 82: 585-593, 2007.

13. Ding D, Yao Y, Yang $C$ and Zhang S: Identification of mannose receptor and CD163 as novel biomarkers for colorectal cancer. Cancer Biomark 21: 689-700, 2018.
14. Dent P: Crosstalk between ERK, AKT, and cell survival. Cancer Biol Ther 15: 245-246, 2014.

15. Nathanson L (ed): Malignant Melanoma: Biology, Diagnosis, and Therapy. Springer US, Boston, MA, 1988.

16. Chung LY, Tang SJ, Sun GH, Chou TY, Yeh TS, Yu SL and Sun KH: Galectin-1 promotes lung cancer progression and chemoresistance by upregulating p38 MAPK, ERK, and cyclooxygenase-2. Clin Cancer Res 18: 4037-4047, 2012.

17. Wang H, Wang D, Li C, Zhang X, Zhou X and Huang J: High $\mathrm{Kpn} \beta 1$ expression promotes non-small cell lung cancer proliferation and chemoresistance via the PI3-kinase/AKT pathway. Tissue Cell 51: 39-48, 2018.

18. Fumarola C, Bonelli MA, Petronini PG and Alfieri RR: Targeting PI3K/AKT/mTOR pathway in non small cell lung cancer. Biochem Pharmacol 90: 197-207, 2014.

19. Vansteenkiste JF, Canon JL, De Braud F, Grossi F, De Pas T, Gray JE, Su WC, Felip E, Yoshioka H, Gridelli C, et al: Safety and efficacy of buparlisib (BKM120) in patients with PI3K pathway-activated non-small cell lung cancer: Results from the phase II BASALT-1 study. J Thorac Oncol 10: 1319-1327, 2015.

20. Tolcher AW, Khan K, Ong M, Banerji U, Papadimitrakopoulou V, Gandara DR, Patnaik A, Baird RD, Olmos D, Garrett CR, et al: Antitumor activity in RAS-driven tumors by blocking AKT and MEK. Clin Cancer Res 21: 739-748, 2015.

21. Deutsch E, Le Péchoux C, Faivre L, Rivera S, Tao Y, Pignon JP, Angokai M, Bahleda R, Deandreis D, Angevin E, et al: Phase I trial of everolimus in combination with thoracic radiotherapy in non-small-cell lung cancer. Ann Oncol 26: 1223-1229, 2015.

22. Blumenschein GR Jr, Gatzemeier U, Fossella F, Stewart DJ, Cupit L, Cihon F, O'Leary J and Reck M: Phase II, multicenter, uncontrolled trial of single-agent sorafenib in patients with relapsed or refractory, advanced non-small-cell lung cancer. J Clin Oncol 27: 4274-4280, 2009.

23. Cairns RA, Harris IS and Mak TW: Regulation of cancer cell metabolism. Nat Rev Cancer 11: 85-95, 2011.

24. de la Fuente M and Hernanz A: Enzymes of mannose metabolism in murine and human lymphocytic leukaemia. Br J Cancer 58: 567-569, 1988.

25. Cazet A, Charest J, Bennett DC, Sambrooks CL and Contessa JN: Mannose phosphate isomerase regulates fibroblast growth factor receptor family signaling and glioma radiosensitivity. PLoS One 9: e110345, 2014.

This work is licensed under a Creative Commons Attribution-NonCommercial-NoDerivatives 4.0 International (CC BY-NC-ND 4.0) License. 